\title{
Neurotensin receptor 1 agonist provides neuroprotection in pre-diabetic rats
}

\author{
Napatsorn Saiyasit ${ }^{1,2}$, Titikorn Chunchai ${ }^{1,2}$, Thidarat Jaiwongkam ${ }^{1,2}$, Sasiwan Kerdphoo ${ }^{1,2}$, Nattayaporn Apaijai ${ }^{1,2}$, \\ Wasana Pratchayasaku|1,2, Jirapas Sripetchwandee1,2, Nipon Chattipakorn ${ }^{1,2}$ and Siriporn C Chattipakorn1,2,3 \\ ${ }^{1}$ Neurophysiology Unit, Cardiac Electrophysiology Research, and Training Center, Faculty of Medicine, Chiang Mai University, Chiang Mai, Thailand \\ ${ }^{2}$ Center of Excellence in Cardiac Electrophysiology, Chiang Mai University, Chiang Mai, Thailand \\ ${ }^{3}$ Department of Oral Biology and Diagnostic Sciences, Faculty of Dentistry, Chiang Mai University, Chiang Mai, Thailand \\ Correspondence should be addressed to S C Chattipakorn: siriporn.c@cmu.ac.th
}

\begin{abstract}
Exogenous treatment of a neurotensin receptor 1 (NTR1) agonist exerted the neuroprotection in an obese and Alzheimer's model. However, the effects of NTR1 modulation on peripheral/hippocampal impairment and cognitive deficit following sustained HFD consumption are poorly understood. Forty rats received a normal diet (ND) or HFD for 16 weeks. At week 13, the ND group received a vehicle $(n=8)$. Thirtytwo HFD-fed group were randomized into four subgroups ( $n=8 /$ subgroup) with a vehicle, $1 \mathrm{mg} / \mathrm{kg}$ of NTR1 agonist, $1 \mathrm{mg} / \mathrm{kg}$ of NTR antagonist, and combined treatment (NTR1 agonist-NTR antagonist) for 2 weeks, s.c. injection. Then, the cognitive tests and peripheral/hippocampal parameters were determined. Our findings demonstrated that NTR1 activator reversed obesity and attenuated metabolic impairment in pre-diabetic rats. It also alleviated hippocampal pathologies and synaptic dysplasticity, leading to deceleration or prevention of cognitive impairment progression. Therefore, NTR1 activation would be a possible novel therapy to decelerate or prevent progression of neuropathology and cognitive impairment in the pre-diabetes.
\end{abstract}
Key Words
- cognition
- high-fat diet
- hippocampus
- insulin resistance
- neurotensin receptor 1
- pre-diabetes

\section{Introduction}

Prolonged a high-fat diet (HFD) intake induces body weight gain, hyperlipidemia, and systemic insulin resistance (Nguyen \& El-Serag 2010, McGillicuddy et al. 2013) which are characteristics of the pre-diabetic condition (Mayans 2015). Long-term ( $\geq 12$ weeks) of HFD consumption has been shown to trigger pathologies in several brain regions, in particular in the hippocampus (Saiyasit et al. 2019), which is associated with spatial learning and memory (Shrager et al. 2007). Previous reports demonstrated that enhanced microglial activation, increased ROS generation, impaired insulin sensitivity, and reduced neuronal synaptic plasticity have been occurred in the hippocampus of HFD-induced pre-diabetic models (Stranahan et al. 2008, Pratchayasakul et al. 2011, Chunchai et al. 2018). These pathologies subsequently potentiated cognitive decline and the development of Alzheimer's disease (Stranahan et al. 2008, Rollins et al. 2019, Saiyasit et al. 2019).

Neurotensin (NT) is an endogenous tridecapeptide synthesized in the neurons and expressed in several brain areas such as the hippocampus, entorhinal cortex, and hypothalamus (Carraway \& Leeman 1973, Satoh \& Matsumura 1990, Rowe et al. 2006). It acts via three types of neurotensin receptors (NTRs), NTR1, NTR2, and NTR3 (Vincent 1995). NTR1 has a crucial role in the modulation of cognition, and satiety (Feifel et al. 2010, Xiao et al. 
2014). The expression of brain NTR1 was elevated in the hippocampus, amygdala, and hypothalamic area in hamsters-treated with HFD (Fazzari et al. 2018). In contrast, the reduction of NT binding site/NTR1 level was displayed in the hippocampus of cognitively impaired rats (Rowe et al. 2006) and postmortem hippocampal tissue of Alzheimer's patients (Jansen et al. 1990). Although current findings regarding the expression of brain NTR1 in association with cognitive decline following HFD-induced obesity and Alzheimer's disease are controversial, the administration of an exogenous NTR1 agonist, which passed directly through the blood-brain barrier, result in attenuation of brain apoptosis/inflammation, regulation of food consumption, and improved cognition in the models of genetic-induced obesity and stroke (Feifel et al. 2010, Keiser et al. 2014, Zhao et al. 2020). The NTR1 agonist also showed synergistic effects with a GLP-1 analog in the restoration of metabolic dysfunction in obese mice (Ratner et al. 2019). Therefore, treatment with an NTR1 agonist may confer benefits on cognition and obesity management in the pre-diabetes. However, there are few studies investigating the effects of an NTR1 agonist on metabolic disturbance, hippocampal pathologies, and cognitive dysfunction following HFDinduced pre-diabetes.

In the current study, we desired to determine the roles of NTR1 modulation using an NTR1 agonist, the NTR antagonist, and the two as a combined therapy on the peripheral/hippocampal pathologies and cognition in sustained HFD-induced pre-diabetic rats. We hypothesized that the treatment with the NTR1 agonist in the HFD-fed group reduces obesity with metabolic disturbance, restores the ileal NTR1 expression, and ameliorates hippocampal pathologies and synaptic dysfunction, eventually restoring cognition through hippocampal NTR1 signaling.

\section{Materials and methods}

\section{Animals and diets}

Male Wistar rats $100-120 \mathrm{~g}(n=40)$ were purchased from Nomura Siam International Co., Ltd. (Bangkok), Thailand and acclimated in a room with temperature, light-darkness cycle, and humidity-monitored environment for 1 week. After that eight rats received a normal diet (ND, 19.77 $\% \mathrm{E}$ fat) and 32 rats received a HFD (59.28 \% fat). During this period, food intake and body weight was determined. At week 13, ND-group received a saline solution, as a vehicle group. HFD-group randomized into four subgroups ( $n=8$ /group) and treated with either the vehicle, PD149163 (NTR1 agonist, $1 \mathrm{mg} / \mathrm{kg}$ ), SR142948A (NTR antagonist,
$1 \mathrm{mg} / \mathrm{kg})$, or the combined therapy of PD149163 (1 mg/kg) and SR142948A (1 mg/kg), total volume $1 \mathrm{~mL}$. Rats were injected subcutaneously for 2 consecutive weeks. Then, the behavioral tests and the oral glucose tolerance test (OGTT) were measured. At week 16, rats were decapitated, liver and ileal tissues were isolated to detect the insulinand NT-related proteins, respectively, the hippocampal tissues were isolated to determine the hippocampal pathologies as shown in Supplementary Fig. 1 (see section on supplementary materials given at the end of this article). The experimental procedures were permitted by the Institutional Ethics committee for Animal Care, Faculty of Medicine, Chiang Mai University (47/2561).

\section{Drug preparation}

PD149163 and SR142948A from Sigma-Aldrich were used in this study. PD149163 was dissolved in 0.9\% NSS and SR142948A was dissolved in $0.9 \%$ NSS at $60^{\circ} \mathrm{C}$. For the combination treatment, SR142948A was injected $30 \mathrm{~min}$ before PD149163 injection. Dosage of PD149163 and SR142948A were chosen in accordance to previous studies (Azmi et al. 2006, Feifel et al. 2010, Keiser et al. 2014).

\section{The biochemical analysis}

After $5 \mathrm{~h}$ of fasting, the tail vein blood was drawn. A colorimetric assay kit (Biotech) was used to examine the levels of plasma glucose. It was also used for triglyceride (TG) and total cholesterol (TC) determination. The levels of high-density lipoprotein cholesterol (HDL-C) and lowdensity lipoprotein cholesterol (LDL-C) were examined using a colorimetric assay kit (Biovision). The sandwich ELISA kit (LINCO Research) was used for insulin determination.

Serum was collected after rats were decapitated to measure the level of malondialdyhyde (MDA) using HPLC method as previously described (Candan \& Tuzmen 2008). The level of serum MDA was computed with the standard curve and shown in $\mu \mathrm{mol} / \mathrm{L}$.

\section{Assessment of insulin resistance}

After the day of the behavioral tests, rats were performed the OGTT. Rats received a glucose solution (2 $\mathrm{g} / \mathrm{kg})$ via oral feeding after they were fasted overnight. Plasma was drawn from tail vein at five time points, specifically $0,15,30,60$, and $120 \mathrm{~min}$. After that an area under the curve of glucose (AUCg) was determined. An index of the HOMA-IR was computed to demonstrate the severity of insulin resistance (Thonusin et al. 2019). 


\section{The behavioral tests}

The open-field test (OFT) was carried out to indicate locomotor activity. A rat was put in the middle of a circular box which had been adapted as explained in a previous report (Denenberg 1969). It was permitted to investigate an empty box for $5 \mathrm{~min}$. Then, the speed and distance were monitored.

The Morris water maze (MWM) test was executed to indicate the hippocampal-dependent memory after the day of OFT. In the training phase (day 1 to day 5), each rat was put into a modified pool with external cues and a hidden platform (Vorhees \& Williams 2006). The rat was given $120 \mathrm{~s}$ to explore the pool and find the platform, four trials/day. After the hidden platform was removed from the target area on day 6 (test phase), the rat was again put in the pool to discover the hidden platform. All parameters were detected using The SMART 3.0 software (Panlab, Harvard Apparatus, Barcelona, Spain).

\section{Level of hippocampal ROS determination}

The level of hippocampal ROS production was determined by incubating tissue samples with fluorescent DCFH-DA (dichloro-hydro fluorescein diacetate) dye as previously described (Pintana et al. 2015). Then, the percentage of changes in the level of hippocampal ROS was calculated.

\section{Immunofluorescence staining for microglial morphology}

The immunostaining process was determined following the procedure described in a previous report (Chunchai et al. 2018). After hippocampal tissues were incubated in $30 \%$ peroxide for $1 \mathrm{~h}$, the antibody of ionized calcium-binding adapter molecule 1 (Iba-1) was applied and incubated overnight in $4^{\circ} \mathrm{C}$. Then, DAPI (Tocris Bioscience, Bristol, UK) and the secondary antibodies of Iba-1 were added. The confocal microscopy (FV3000, Olympus Corp, Tokyo, Japan) and Imaris software 7.0 (Bitplane, Oxford instrument company, AG, Zurich, Switzerland) were used to analyze the data.

\section{Terminal deoxy nucleotidyl nick-end labeling staining}

Hippocampal slices $15 \mu \mathrm{m}$ thick were incubated with Proteinase $\mathrm{k}$ solution $(1: 50,50 \mu \mathrm{L})$ and Cytonin $(50 \mu \mathrm{L})$ for 30 and 120 min, respectively (Keawtep et al. 2019). The TACS nuclease buffer containing TACS nuclease (1:50) was used as a positive control. Then, an inverted microscope (Nikon) and Image J (NIH image) software were used to analyze the data.

\section{Preparation of proteins for immunoblotting}

To determine the level of insulin-associated proteins, the hippocampal slices were incubated with either aCSF or aCSF with $500 \mathrm{nM}$ of insulin (Humulin R, Eli Lilly, Giessen, Germany). They were homogenized in the lysis buffer as previously described (Pratchayasakul et al. 2011). To measure the expression of other proteins in the liver and hippocampus, tissues were homogenized with $1 \mathrm{~mL}$ of non-ionizing lysis buffer. The concentration of the proteins was determined by the Bio-Rad DC Protein assay kit (Bio-Rad Laboratories). All samples were preserved at $-80^{\circ} \mathrm{C}$ until analysis.

\section{Immunoblotting}

Immunoblotting was conducted in line with conventional electrophoresis methods as described previously (Kumfu et al. 2018). After transferring the proteins onto nitrocellulose membranes, the membranes were incubated with primary antibodies against NT signaling: NTR1, total phosphokinase C (PKC), and phospholipase C beta-1 (PLC $\beta-1)$, insulin receptor substrate 1 (IRS1), phosphorylation of IRS1 at serine 307 (p-IRS1 ${ }^{\text {ser307}}$ ), Akt, phosphorylation of Akt at serine 473 (p-Akt ${ }^{\text {ser473)}}$, AMPK, phosphorylation of AMPK $\alpha$ (Thr172) (p-AMPK), Bax, Bcl-2 and loading controls: GAPDH and $\beta$-actin overnight at $4^{\circ} \mathrm{C}$. The horseradish peroxidaseconjugated anti-rabbit secondary antibodies were added for $1 \mathrm{~h}$ followed by ECL Western blotting substrate. ChemiDoc Touch Imaging System (Bio-Rad Laboratories) and Image J (NIH image) software was used to analyze the band intensity.

\section{Long-term potentiation (LTP) recording}

The preparation of hippocampal slices were shown in a previous report (Pratchayasakul et al. 2011). After a bipolar electrode was put at the Schaffer collateralcommissural pathway, high-frequency stimulation (HFS, 4 series at $100 \mathrm{~Hz}$; $0.5 \mathrm{~s}$ duration; $20 \mathrm{~s}$ interval) was applied. The CA1 region was chosen to determine the field excitatory postsynaptic potentials (fEPSPs). The percentage increment of fEPSP slopes after HFS was analyzed by pClamp10.6 software (Axon Instruments, CA, USA). 


\section{Dendritic spine density}

The hippocampal spine density was determine using the Golgi staining solutions (FD Neurotechnologies kit, PK 401, Ellicott City, USA). After an immunostaining process, the CA1 area was chosen to determine the tertiary branches of the dendritic spines. An inverted microscope (IX-81, Olympus, Tokyo, Japan) and Xcellence software (Olympus, Tokyo, Japan) were used to analyze the findings.

\section{Statistical analysis}

Mean \pm S.E.M. was used to express all of the findings. A repeated two-way ANOVA with Fisher's LSD analysis was used to test the significance of the data from the training phase. A one-way ANOVA with Fisher's LSD analysis was used for multiple comparisons. Statistical significance was accepted as $P<0.05$.

\section{Results}

The activation of NTR1 restored energy intake and ileal NT-related protein as well as alleviated hepatic/ systemic insulin resistance, but did not reduce hyperlipidemia and systemic oxidative stress in HFD-induced pre-diabetic rats

Sixteen weeks of HFD-fed rats showed a marked increase in body weight, visceral fat, level of plasma LDL-C, total cholesterol, and serum MDA but did not alter food intake, levels of plasma triglyceride and HDL-C, compared to ND-fed group (Supplementary Fig. 2A, B, C, D, E, F, $G$ and $H)$. An enhancement in the level of NTR1 and the reduction in the level of p-AMPK, the downstream signaling of ileal NT/NTR1 were detected in HFD-fed rats, compared to ND-fed rats (Fig. 1A, B and C). Also, the hepatic insulin pathway including the expression of IRS1 and p-Aktser473/Akt were reduced in the HFDfed rats, compared to ND-fed rats (Fig. 2A, B and C). Furthermore, high levels of plasma AUCg, insulin, and HOMA index without any change in the level of fasting blood glucose were observed in the HFD-fed rats treated with vehicle, in comparison to the ND-fed rats (Fig. 2D, E, F and G). Altogether, these results proposed that an HFD for 16 weeks in rats developed a pre-diabetes, as indicated by obesity, hyperlipidemia, and hepatic/ systemic insulin resistance, as well as the alteration of ileal NT-related protein.

Treatment with PD149163 in HFD rats reduced body weight, visceral fat, and food intake. However, there were no changes in these parameters from HFD-fed rats treated with vehicle and SR142948A. Interestingly, body weight, visceral fat, and food intake in HFD-fed rats treated with the combined therapy showed a similar pattern to that in HFD-fed rats treated with the NTR1 agonist alone (Supplementary Fig. 2A, B and C). However, the administration of PD149163, SR142948A, and the two combined in HFD-fed rats did not affect lipid profiles and the level of serum MDA, when compared to HFD-fed group (Supplementary Fig. 2D, E, F, G and H).
A

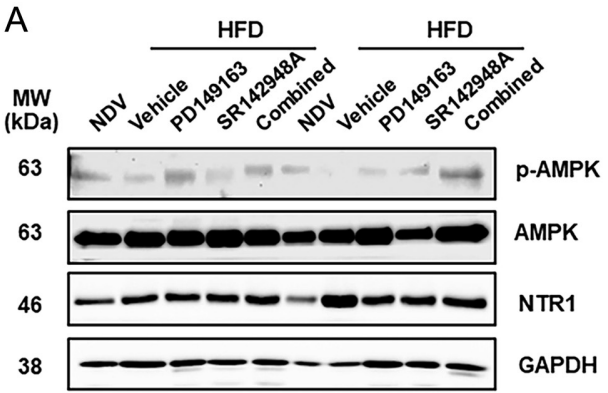

C

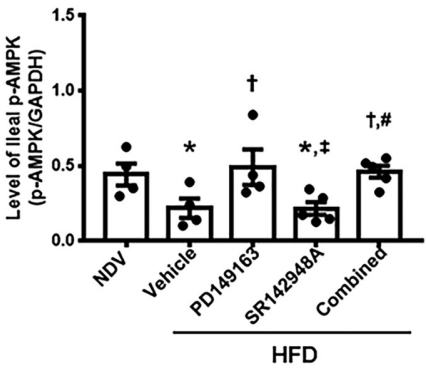

B

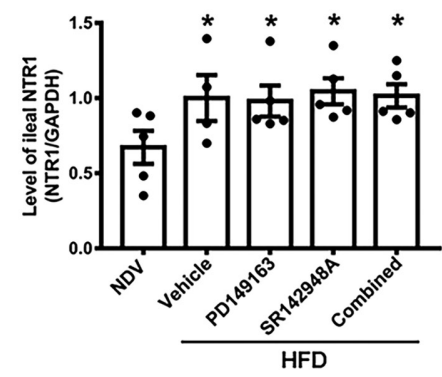

D

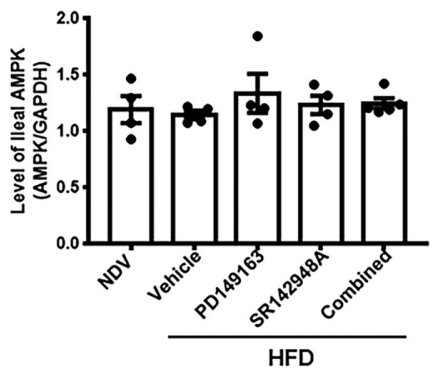

\section{Figure 1}

Effects of NTR1 modulation on ileal NTR1 and its related protein in HFD-induced pre-diabetic rats ( $n=4-6 /$ group). (A) The representative bands of NT signaling in the ileum which are the expression of NTR1, p-AMPK, AMPK; (B) the protein level of ileal NTR1; (C) the protein level of ileal p-AMPK; and (D) the protein level of ileal AMPK. HFD, high-fat diet; NDV, normal diet-fed rats treated with vehicle; NTR1, neurotensin receptor 1 . $\star P<0.05$ vs ND-fed rats treated with vehicle; ${ }^{+} P<0.05$ vs HFD-fed rats treated with vehicle; $\ddagger P<0.05$ vs HFD-fed rats treated with PD149163; $\# P<0.05$ vs HFD-fed rats treated with SR142948A. 
A

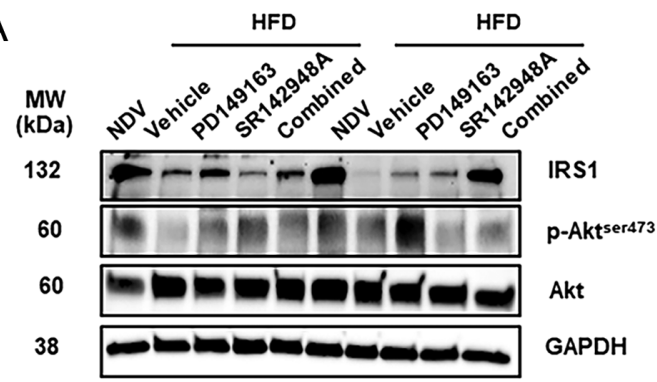

$\mathrm{D}$

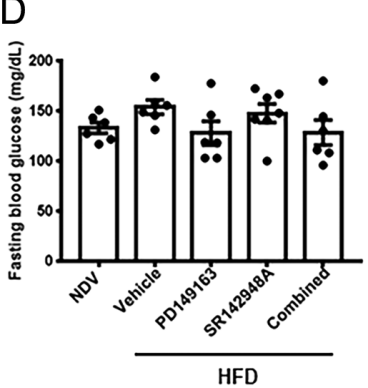

B
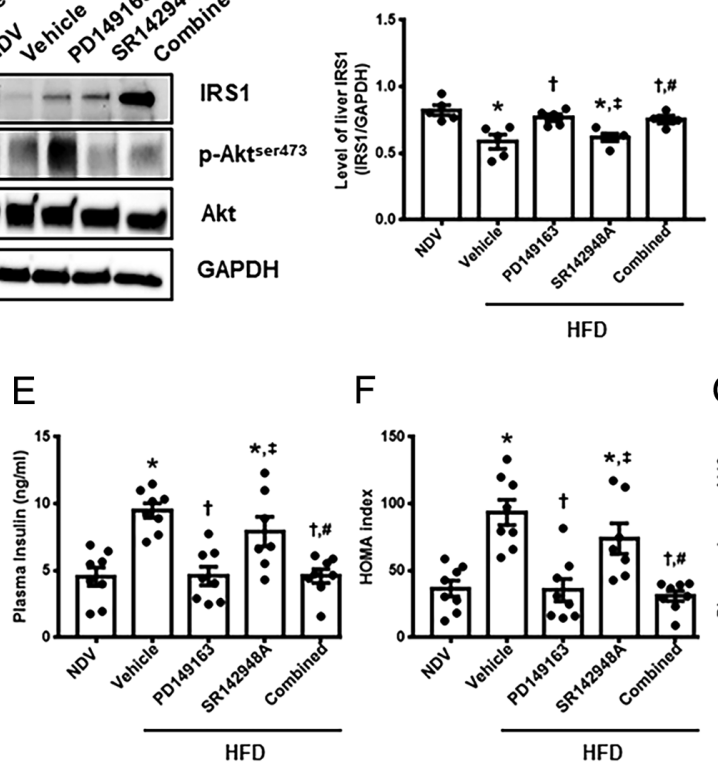

$\mathrm{F}$

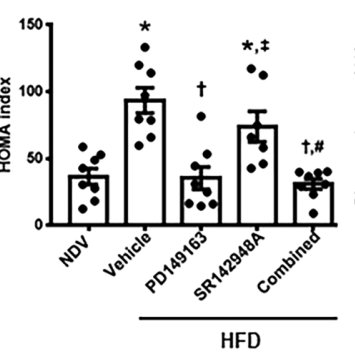

C

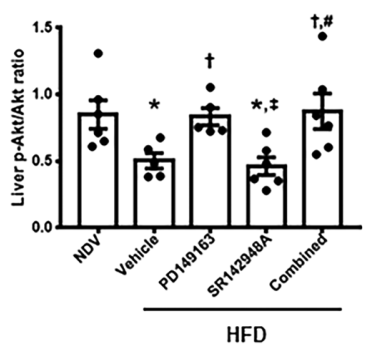

G

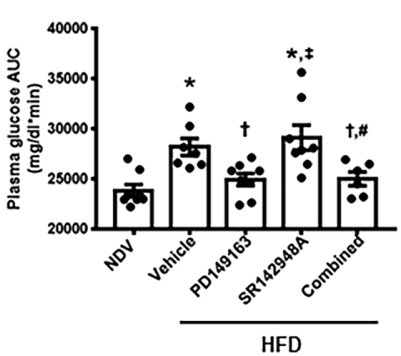

Figure 2

Effects of NTR1 modulation on the hepatic and systemic insulin signaling in HFD-induced pre-diabetic rats ( $n=6-8 /$ group). (A) The representative bands of insulin signaling in the liver which are IRS1, p-Aktser473, and Akt; (B) the level of liver IRS1; (C) the ratio of p-Aktser473/Akt in the liver; (D) plasma glucose AUC from OGTT; (E) plasma insulin; (F) HOMA index; (G) fasting blood glucose. AUC, area under the curve; HFD, high-fat diet; HOMA, homeostatic model assessment; IRS1, insulin receptor substrate 1; ND, normal diet; OGTT, oral glucose tolerance test; p-Aktser473, phosphorylation of Akt at serine 473. ${ }^{\star} P<0.05$ vs ND-fed rats treated with vehicle; ${ }^{\dagger} P<0.05$ vs HFD-fed rats treated with vehicle; ${ }^{*} P<0.05$ vs HFD-fed rats treated with $\mathrm{PD} 149163 ;{ }^{\#} P<0.05$ vs HFD-fed rats treated with SR142948A.

Although the expression of ileal NTR1 did not change after treated with NTR1 modulators, the level of ileal p-AMPK was augmented in HFD-treated with PD149163, compared to HFD-fed rats treated with vehicle and SR142948A (Fig. 1A, B and C). The result in the combined group showed the similar pattern with HFD treated with PD149163. Notably, the administration of PD149163 attenuated hepatic/systemic insulin insensitivity in HFD-fed rats by enhancing the expression of IRS1 and p-Aktser473/Akt in the liver, reducing the level of plasma AUCg, decreasing the level of plasma insulin, and decreasing the HOMA index when compared to HFDfed rats treated with vehicle (Fig. 2A, B, C, D, E, F and $\mathrm{G})$. Contrarily, they were unchanged in these parameters between HFD-fed rats treated with SR142948A and vehicle. Surprisingly, the results in the combined group showed a similar pattern to HFD-fed rats treated with PD149163 (Fig. 2D, E, F and G). Altogether, these findings indicate that the NTR1 activator restored energy intake, the activity of ileal NT-related protein, and hepatic/systemic insulin insensitivity but did not affect the circulating lipid profiles and oxidative stress in HFD-induced pre-diabetes. However, these beneficial effects of the NTR1 agonist were not blocked by the NTR antagonist, suggesting that the effects of NTR1 agonist on the metabolic parameters of HFD-induced pre-diabetic rats might be occurred in a receptor-independent manner.

\section{The activation of NTR1 enhanced the activity of hippocampal NTR1 signaling in HFD-induced pre-diabetic rats}

To confirm the role of the NTR1 agonist in hippocampal NTR1 signaling, the protein levels of hippocampal NTR1, PKC, and PLC $\beta-1$ were observed. Although these parameters were unchanged in both HFD- and ND-fed rats treated with vehicle, treatment of PD149163 effectively enhanced the expression of hippocampal PLC $\beta-1$ and PKC, when compared with HFD-fed rats (Fig. 3A, B, C and $\mathrm{D})$. However, these proteins were not significantly different in HFD-fed rats treated with SR142948A and the combined therapy, in comparison to those of HFDfed rats treated with vehicle (Fig. 3A, B, C and D). Taken together, the exogenous administration of NTR1 agonist in HFD-fed rats modulated the downstream signaling of hippocampal NTR1. 


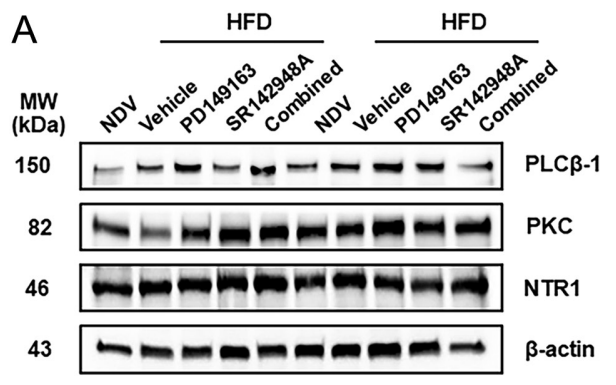

C

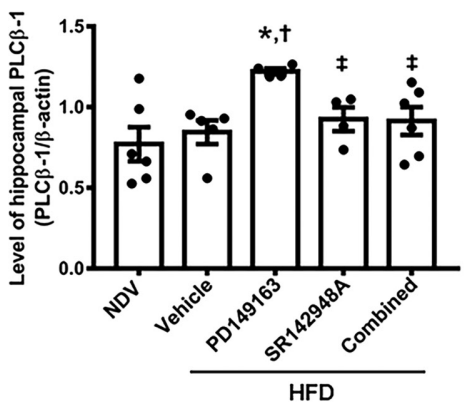

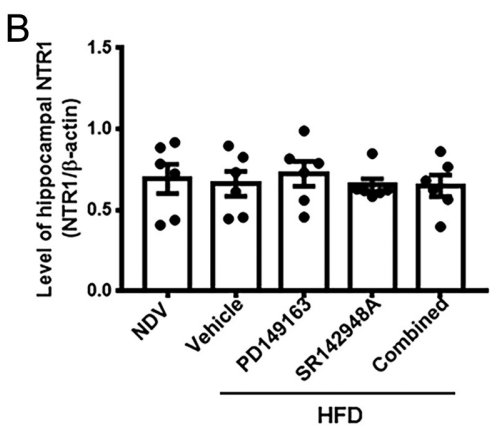

D

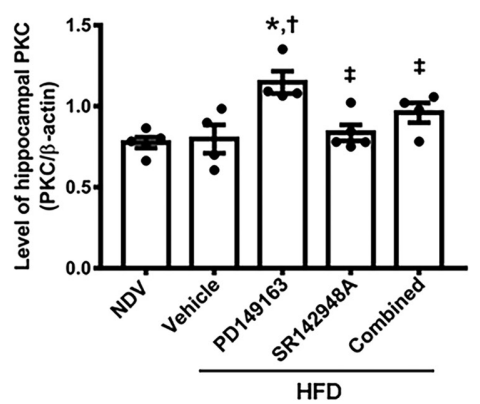

Figure 3

Effects of NTR1 modulation on the activity of hippocampal NTR1 signaling in HFD-induced pre-diabetic rats ( $n=4-6 /$ group). (A) The representative bands of hippocampal NTR1 signaling which are NTR1, PLC- $\beta 1$, and PKC; (B) the protein level of hippocampal NTR1; (C) the protein level of hippocampal PLC $\beta-1$; and (D) the protein level of hippocampal PKC. HFD, high-fat diet; NDV, ND-fed rats treated with vehicle; NTR1,

neurotensin receptor 1 ; PKC, protein kinase $C$; PLC- $\beta 1$, phospholipase $C$ beta- $1 . * P<0.05$ vs ND-fed rats treated with vehicle; ${ }^{+} P<0.05$ vs HFD-fed rats treated with vehicle; $¥ P<0.05$ vs HFD-fed rats treated with PD149163.
The activation of NTR1 ameliorated hippocampal insulin insensitivity in HFD-induced pre-diabetic rats

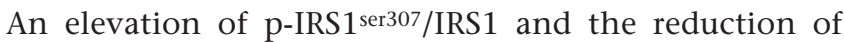

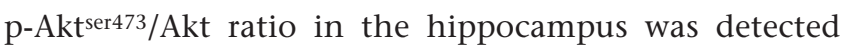
in the HFD-fed rats, in comparison to the ND-fed rats (Fig. 4A, B, C, D, E and F), implying hippocampal insulin insensitivity. Notably, administration of PD149163 in HFD-fed rats returned to within normal limits in these two parameters. The application of SR142948A and combined therapy in HFD-fed rats showed similar findings to those in the HFD-fed rats treated with vehicle (Fig. 4A, B, C, D, E and F). These results demonstrate that chronic HFD consumption caused hippocampal insulin insensitivity, which was attenuated by the activation of NTR1.

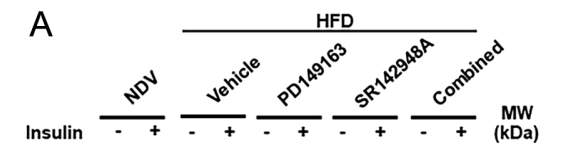

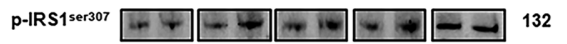

IRS1 -200 GAPDH 38

$\mathrm{B}$

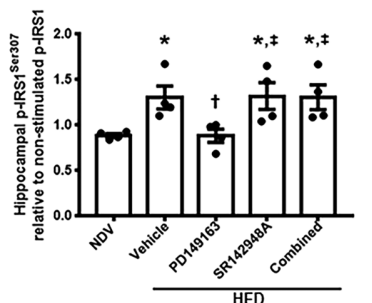

C

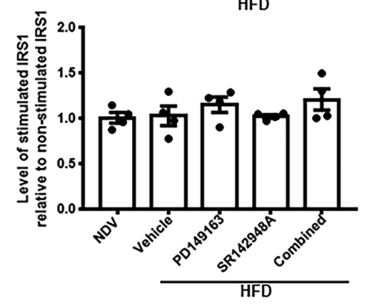

D
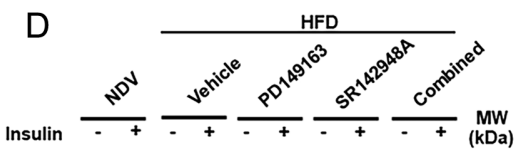

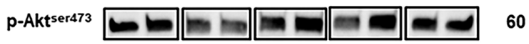

Akt 60 GAPDH 38

E

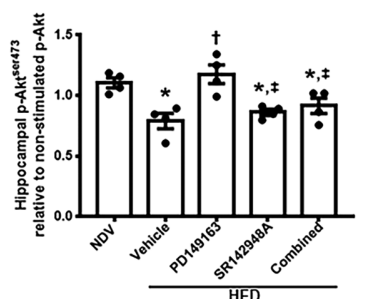

$\mathrm{F}$

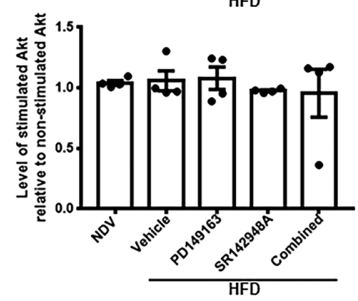

\section{Figure 4}

Effects of NTR1 modulation on hippocampal insulin sensitivity after incubation with insulin in HFD-induced pre-diabetic rats ( $n=4 /$ group). (A) The representative bands of hippocampal p-IRS1 1 er307 and IRS1; (B) the protein level of hippocampal p-IRS1 1 ser307 relative to nonstimulated p-IRS1; (C) the protein level of stimulated IRS1 to non-stimulated IRS1; (D) the representative bands of hippocampal $\mathrm{p}$-Aktser473 and Akt; (E) the protein level of hippocampal p-Aktser473 relative to non-stimulated p-Akt; (F) the protein level of stimulated Akt to non-stimulated Akt. IRS1, insulin receptor substrate 1; HFD, high-fat diet; NDV, ND-fed rats treated with vehicle; $p$-Aktser473, phosphorylation of Akt at serine 473; p-IRS1 ${ }^{\text {ser } 307}$, phosphorylation of insulin receptor substrate 1 at serine 307. $* P<0.05$ vs ND-fed rats treated with vehicle; ${ }^{\dagger} P<0.05$ vs HFD-fed rats treated with vehicle; $¥ P<0.05$ vs HFD-fed rats treated with PD149163. 
The activation of NTR1 re-established hippocampal synaptic plasticity in HFD-induced pre-diabetic rats

Next, the hippocampal synaptic function (the extracellular LTP) was recorded. The percentage increment of fEPSP slopes was diminished in HFD-fed rats, compared with ND-fed rats (Fig. 5A, B and F). After treatment with PD149163, the HFD-fed rats showed a remarkable increase in fEPSP slopes in compared with the HFD-fed rats treated with vehicle. However, this parameter was diminished in HFD-fed rats treated with SR142948A and the combined group, a similar finding to that in HFD-fed group (Fig. 5A, $\mathrm{B}, \mathrm{C}, \mathrm{D}, \mathrm{E}$ and $\mathrm{F}$ ). Thus, sustained HFD consumption caused hippocampal synaptic dysplasticity, which was reduced by the activation of NTR1.

\section{The activation of NTR1 reduced hippocampal spine loss in HFD-induced pre-diabetic rats}

The number of the hippocampal dendritic spines was reduced in HFD-fed rats, in compared with the ND-fed rats (Fig. 6A and B). Notably, treatment with PD149163 in HFD-fed rats elevated this parameter, compared with HFD-fed group. This restorative effect was eliminated after treatment with SR142948A and the combined therapy (Fig. 6A and B). Thereby, the restoration in the number of hippocampal spine density after chronic HFD intake occurred through NTR1 activation.

\section{The activation of NTR1 reduced the number of hippocampal microglial cells in HFD-induced pre-diabetic rats}

Long-term HFD intake for 16 weeks enhanced the number of hippocampal Iba- 1 positive cells, a surface marker of microglia, in comparison to ND-fed rats (Fig. 7A and B). However, there were no changes in Iba-1 positive process length, Iba- 1 positive cell volume, AUC of Iba-1 intersections, and the number of Iba- 1 intersections between HFD- and ND-fed rats treated with vehicle (Fig. 7C, D, E and F). The application of PD149163 in
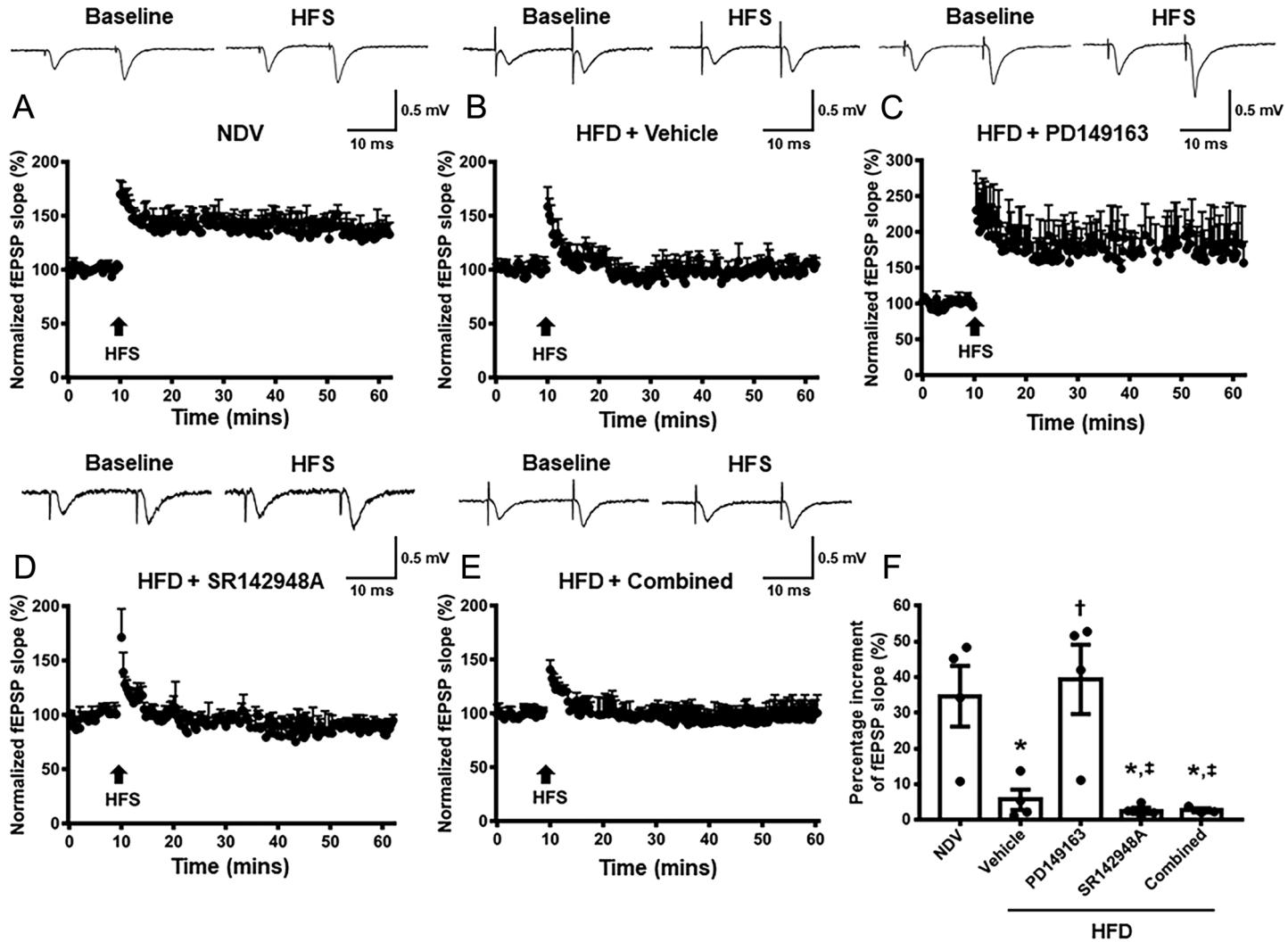

Figure 5

Effects of NTR1 modulation on hippocampal synaptic plasticity in HFD-induced pre-diabetic rats ( $n=3-4$ brain slices/group). (A) The representative tracing and the percentage of the normalized fEPSP slopes in NDV group; (B) HFD-fed rats treated with vehicle; (C) HFD-fed rats treated with PD149163; (D) HFD-fed rats treated with SR142948A; and (E) HFD-fed rats treated with combined drugs. (F) The increment of fEPSP slope. fEPSP, field excitatory postsynaptic potential; HFD, high-fat diet; HFS, high-frequency stimulation; NDV, normal diet-fed rats treated with vehicle. ${ }^{*} P<0.05$ vs ND-fed rats treated with vehicle; ${ }^{\dagger} P<0.05$ vs HFD-fed rats treated with vehicle; ${ }^{\uparrow} P<0.05$ vs HFD-fed rats treated with PD149163. 
A
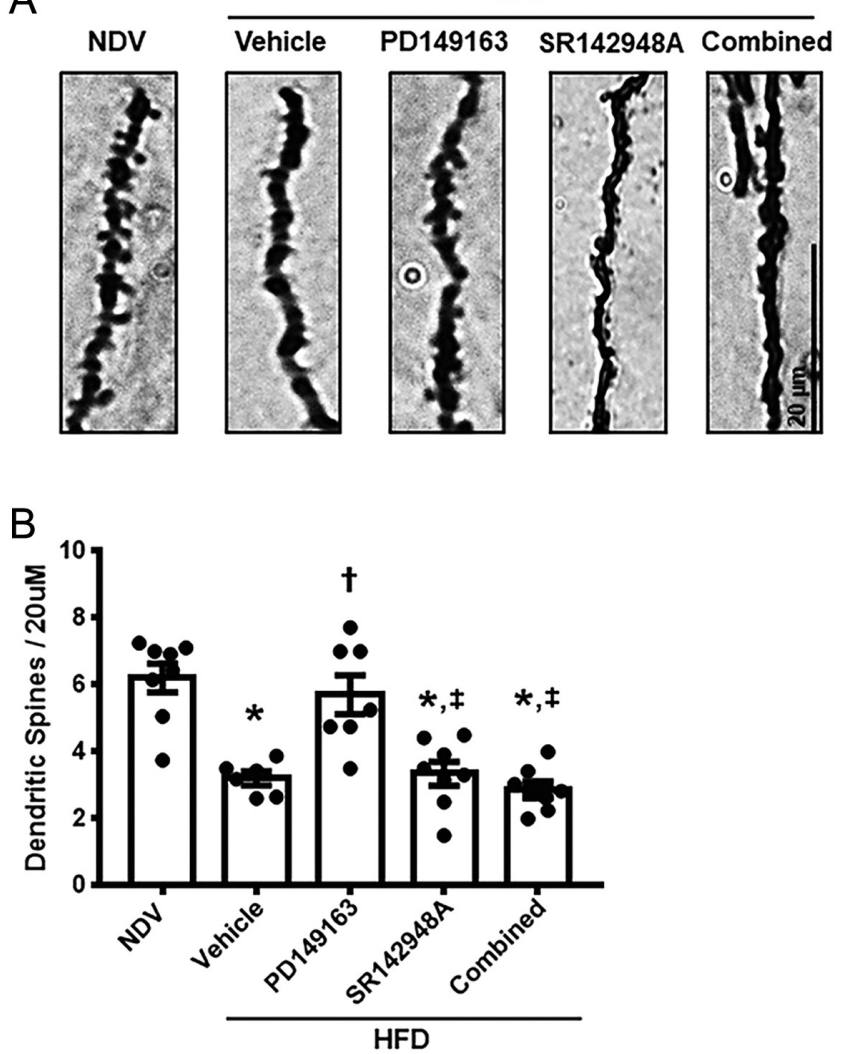

Figure 6

Effects of NTR1 modulation on hippocampal dendritic spine density in HFD-induced pre-diabetic rats ( $n=4-6$ brain slices/group). (A) The representative pictures of dendritic spine density (bar $=20 \mu \mathrm{m})$; (B) the number of dendritic spines in the CA1 region of the hippocampus. HFD, high-fat diet; NDV, normal diet-fed rats treated with vehicle. ${ }^{*} P<0.05$ vs ND-fed rats treated with vehicle; ${ }^{\dagger} P<0.05$ vs HFD-fed rats treated with vehicle; $¥ P<0.05$ vs HFD-fed rats treated with PD149163.

HFD-fed rats decreased hippocampal Iba-1 positive cells and these results were reversed in HFD-fed rats treated with either SR142948A or the combined therapy (Fig. 7A and $B)$. These findings imply that long-term HFD exposure resulted in hippocampal microglial hyper-activation, which was restored by the activation of NTR1.

\section{The activation of NTR1 attenuated hippocampal oxidative stress and apoptosis in HFD-induced pre-diabetic rats}

Additionally, high levels of hippocampal ROS generation, hippocampal TUNEL positive cells, and hippocampal Bax/Bcl-2 expression were observed in HFD-fed rats in comparison with ND-fed rats (Fig. 8A, B, C and D). This suggested that the sustained HFD exposure caused hippocampal oxidative stress and apoptosis. After treatment with PD149163 in HFD-fed group, the level of ROS production, the number of TUNEL positive cells, and the protein level of $\mathrm{Bax} / \mathrm{Bcl}-2$ in the hippocampus were remarkably reduced, in comparison to HFD-fed rats treated with vehicle. Nonetheless, all these parameters were returned to within normal limit following treatment with SR142948A and combined therapy (Fig. 8A, B, C and $\mathrm{D})$, indicating that the attenuation of hippocampal oxidative stress and apoptosis occurred through NTR1 activation in HFD-induced pre-diabetes.

\section{The activation of NTR1 ameliorated cognitive impairment in HFD-induced pre-diabetic rats}

There were no differences in both speed and distance in the locomotor activity analysis as determined by OFT in all groups (Fig. 9A and B). Additionally, the hippocampaldependent memory was tested using the MWM test. In the training period of this test, the time taken to reach the platform was increased in HFD-fed rats, in comparison to ND-fed rats from day 2 to day 5 (Fig. 9D, E, F and G). Also the time spent in the target quadrant from the probe test on day 6 was markedly decreased in HFD-fed rats, in comparison to ND-fed rats (Fig. 9H and I). These results suggest that HFD-induced pre-diabetic rats developed impairment of cognition.

Surprisingly, the HFD-fed rats treated with PD149163 not only showed a reduced time to reach the platform from day 2 to day 5 but also spent a longer time in the target quadrant in comparison to HFD-fed group. In contrast, these parameters were not changed in HFD-fed rats treated with SR142948A and combined therapy, when compared to HFD-fed rats treated with vehicle (Fig. 9D, E, F, G, H and I). Collectively these findings show that the NTR1 agonist ameliorated the cognitive deficits in HFDinduced pre-diabetic rats.

\section{Discussion}

The main highlights in the current study are as follows: (1) Sustained HFD intake contributed to a pre-diabetic condition, as indicated by obesity with hyperlipidemia, hepatic/systemic insulin resistance, and the disruption of ileal NT-related protein; (2) It also caused hippocampal pathologies by inducing microglial hyper-activation, oxidative stress, apoptosis, insulin insensitivity, synaptic dysplasticity, and dendritic spines loss, which resulted in cognitive decline; and (3) treatment with NTR1 activator in pre-diabetic rats exerted beneficial effects on the aforementioned parameters mediated through 


\begin{tabular}{|l|l|l|l|l|}
$\begin{array}{l}\text { Journal of } \\
\text { Endocrinology }\end{array}$ & N Saiyasit et al. & $\begin{array}{l}\text { NTR1 exerted neuroprotection } \\
\text { in pre-diabetes }\end{array}$ & $\mathbf{2 4 8 : 1}$ & $\mathbf{6 7}$ \\
\hline
\end{tabular}

A

HFD
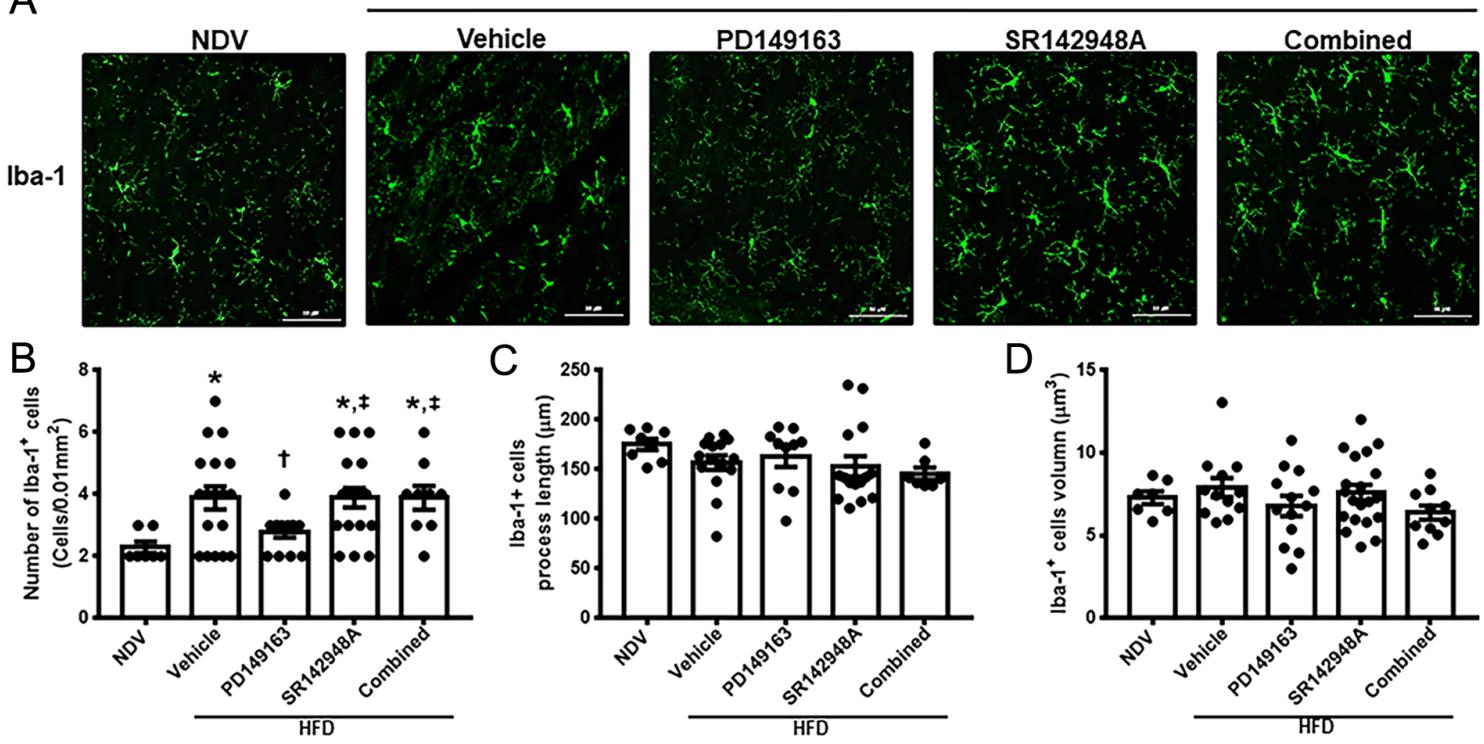

$\mathrm{D}$

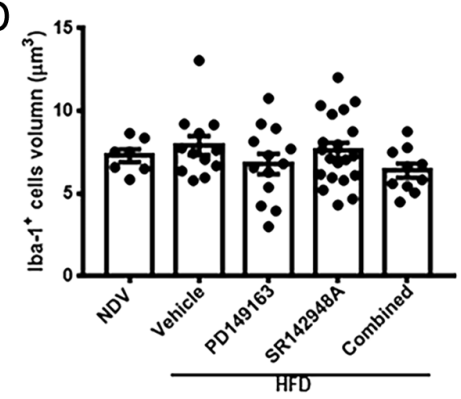

$\mathrm{E}$

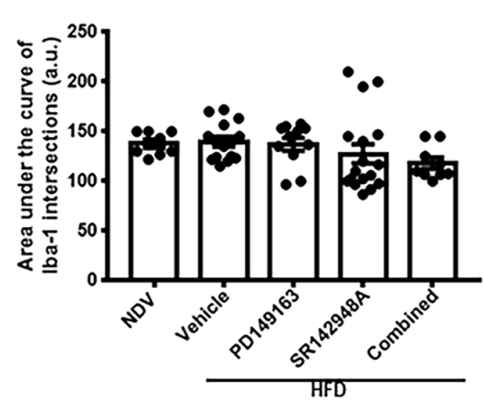

$\mathrm{F}$

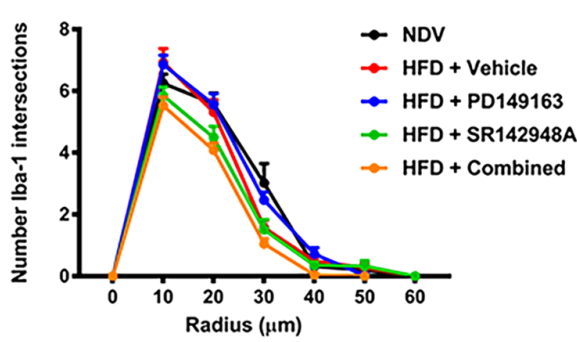

\section{Figure 7}

Effects of NTR1 modulation on microglial morphology in HFD-induced pre-diabetic rats ( $n=4-6$ brain slices/group). (A) The representative images of Iba-1 positive cells in the CA1 region of the hippocampus (bar =50 $\mu \mathrm{m}$ ); (B) the number of Iba-1 positive cells; (C) the process length of Iba-1 positive cells; (D) the Iba-1 positive cell volume; (E); an area under the curve of Iba-1 intersections; and (F) the number of Iba-1 intersections. HFD, high-fat diet; Iba-1, ionized calcium-binding adapter molecule-1; NDV, ND-fed rats treated with vehicle. $* P<0.05$ vs ND-fed rats treated with vehicle; ${ }^{\dagger} P<0.05$ vs HFD-fed rats treated with vehicle; $¥ P<0.05$ vs HFD-fed rats treated with PD149163. A full color version of this figure is available at https://doi.org/10.1530/JOE-20-0439.

the modulation of NTR1 and insulin signaling in the hippocampus as illustrated in Fig. 10.

In this study, the beneficial effects of the NTR1 agonist on peripheral parameters were not blocked by the NTR antagonist, suggesting that the effects of NTR1 agonist on the metabolic parameters of HFD-induced pre-diabetic rats might occur in a receptor-independent manner. However, the activity of NTR1 agonist on other receptors in visceral organs is poorly understood. Therefore, using the NTR1-KO model or the blockers of other NTR subtypes should be further investigated. In addition, a possible explanation for the reduction of body weight and visceral fat in the present study may be via the activation of NTR1 with an NTR1 agonist on the hypothalamic nuclei, resulting in promoting the satiety effect (Sahu 2008, Ratner et al. 2019). In accordance with those previous findings, our results showed markedly decreased food intake, body weight, and visceral fat in HFD-rats treated with the NTR1 agonist, effects which were reversed after the rats received the NTR antagonist. Therefore, NTR1 activation reduced obesity through the regulation of food intake following chronic HFD exposure. Previous studies reported that, besides the beneficial effects of NTR1 agonist, the caloric restriction also attenuate the peripheral pathologies through the reduction of body weight gain and visceral fat in HFDinduced pre-diabetic model (Pintana et al. 2016, Tanajak et al. 2017). Nonetheless, direct comparison between the decreased food intake achieved by caloric restriction and NTR1 modulator is yet to be addressed in future studies.

However, the administration of NTR1 agonist did not affect hyperlipidemia in HFD-fed rats. The possible explanation might be the persistent systemic oxidative stress following long-term HFD consumption. To support this possibility, we measured the level of serum 
A

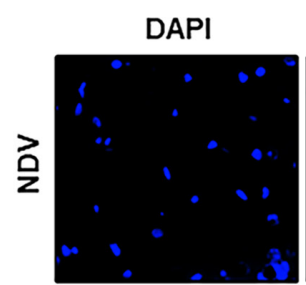

$\mid \frac{0}{\frac{0}{2}}$

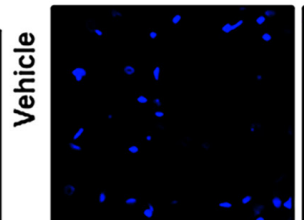

뭄

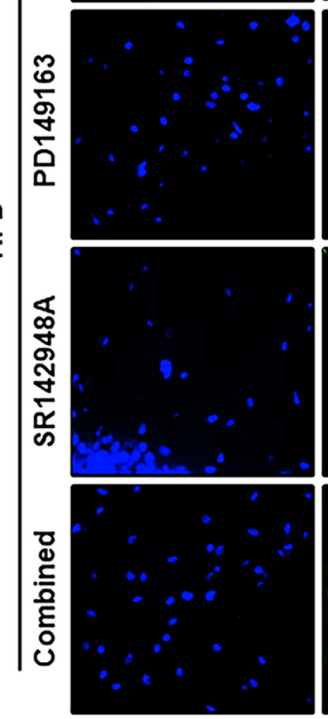

TUNEL
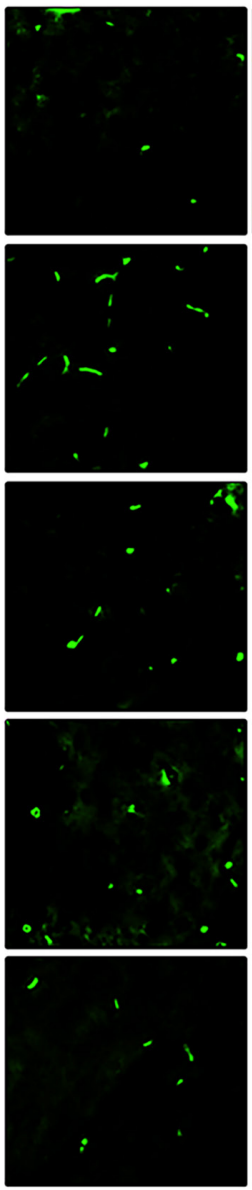
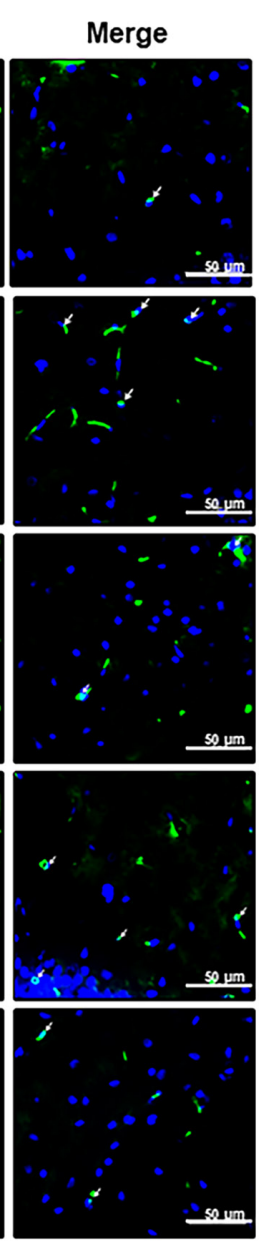

B

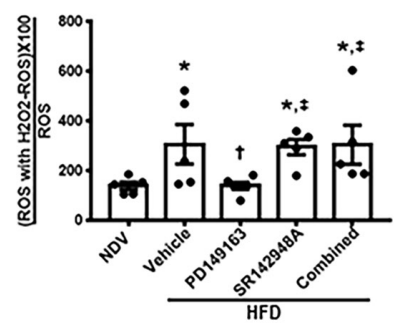

C
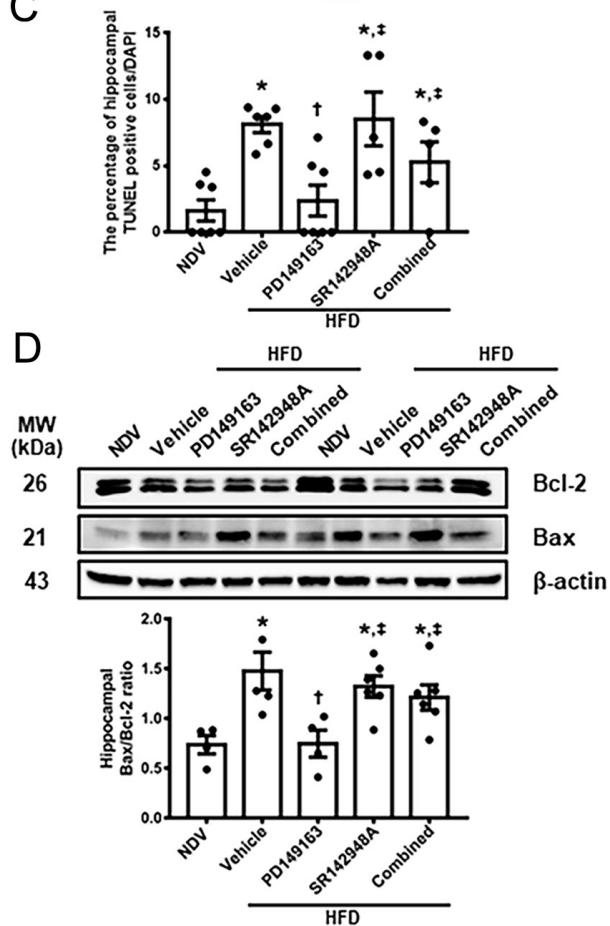

Figure 8

Effects of NTR1 modulation on hippocampal oxidative stress and apoptotic cell death in HFD-induced pre-diabetic rats $(n=4-6 / g r o u p)$. (A) The representative pictures of hippocampal cell death $(\mathrm{bar}=50 \mu \mathrm{m})$; (B) the level of hippocampal ROS production; (C) the percentage of hippocampal TUNEL positive cells/DAPI; and (D) the representative bands and the expression of hippocampal Bax/BCl-2. HFD, high-fat diet; ND, normal diet; NDV: normal diet-fed rats treated with vehicle; ROS, reactive oxygen species. ${ }^{*} P<0.05$ vs ND-fed rats treated with vehicle; ${ }^{\dagger} P<0.05$ vs HFD-fed rats treated with vehicle; $¥ P<0.05$ vs HFD-fed rats treated with PD149163. A full color version of this figure is available at https://doi.org/10.1530/JOE-20-0439.

malondialdehyde (MDA), the biomarker of systemic oxidative stress, and found that the MDA level was increased in HFD-fed rats when compared with ND-fed rats. Treatment with NTR1 agonist, NTR1 antagonist, and combined therapy in HFD-fed rats did not alter the level of serum MDA, when compared with HFD-fed control group (as shown in Supplementary Fig. 2H). This finding is in line with a clinical report demonstrating that a high level of circulating MDA is associated with the progression of hyperlipidemia (Yang et al. 2008). Our previous study reported that although the level of triglyceride was unchanged in the blood, it was increased in the liver and adipose tissue after the rats were fed with HFD for 12 to 16 weeks (Pratchayasakul et al. 2011, 2014). Additionally, the level of plasma HDL-C was not altered after chronic HFD consumption in this study; it might be that the duration

(C) 2021 Society for Endocrinology Published by Bioscientifica Ltd. Printed in Great Britain of HFD intake was not long enough to alter the level of HDL-C (Jinawong et al. 2020). In the present study, the rats were fed with HFD for total 16 weeks; however, another study from our group has reported that the reduction in plasma HDL-C levels could be achieved after 40 weeks of HFD consumption (Saiyasit et al. 2019).

In vivo studies demonstrated that a high level of gut NTR1 was detected following HFD consumption which led to the reduction in the level of p-AMPK, resulting in enhanced fat absorption and accumulation ( $\mathrm{Li}$ et al. 2016, Saiyasit et al. 2020). Even though treatment with NTR1 agonist did not alter the level of ileal NTR1 in this study, the level of ileal p-AMPK was increased, which was reversed in HFD-treated with the NTR antagonist. These results suggest that the activity of ileal NT depends upon NTR1 regulation. Apart from the restoration of ileal 
A
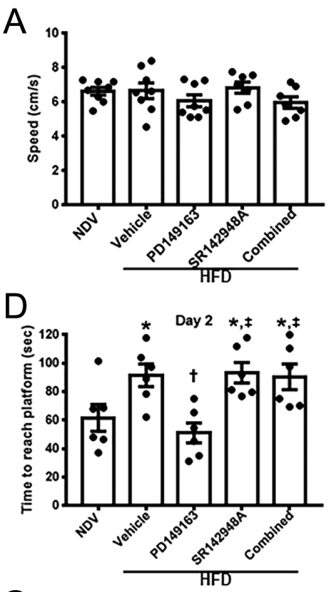

G

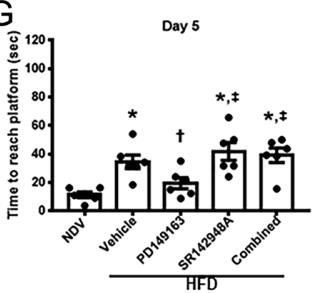

B

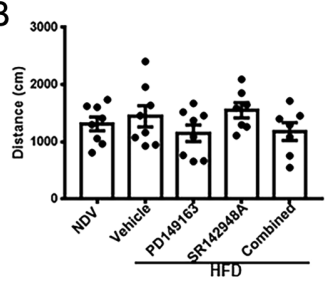

E

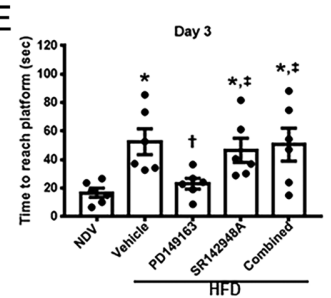

$H$

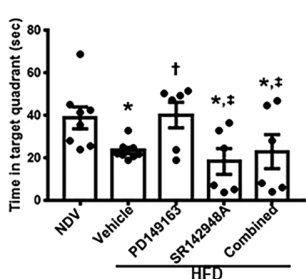

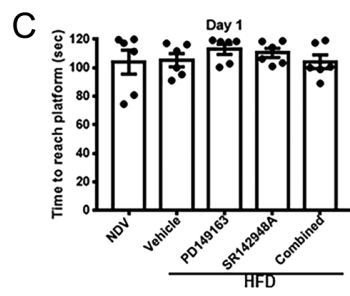

F

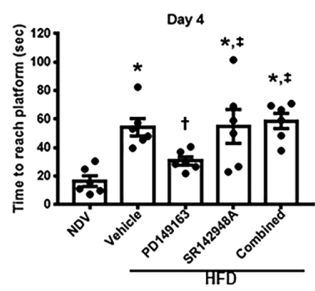

I

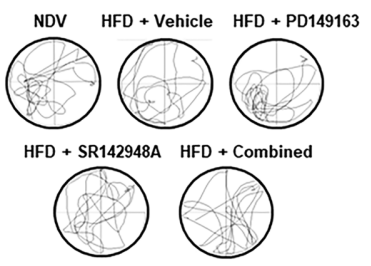

Figure 9

Effects of NTR1 modulation on behavioral tests in HFD-induced pre-diabetic rats ( $n=6-8$ /group). Locomotor activity as demonstrated by (A) speed and (B) distance from OFT. The hippocampaldependent memory as demonstrated by time to reach the platform in the acquisition phase on day 1 to day 5 (C, D, E, F and G); time spent in the target quadrant in the probe test on day $6(\mathrm{H})$; and the representative pictures of direction in the probe test from MWM test (I). HFD, high-fat diet; MWM, Morris water maze; NDV, normal diet-fed rats treated with vehicle; OFT, open-field test. $\star P<0.05$ vs ND-fed rats treated with vehicle; ${ }^{+} P<0.05$ vs HFD-fed rats treated with vehicle; $\ddagger P<0.05$ vs HFD-fed rats treated with PD149163.
NT-related protein, the NTR1 activator also showed the advantageous effects on insulin signaling. Using an NTR1 agonist alleviated peripheral insulin resistance possibly via the reduction of the amount of adiposity in HFD-fed mice (Denver et al. 2018). The co-release of NT, peptide-Y (PYY), and glucagon-like peptide 1 (GLP-1) were detected by L-cells in the small intestine, implying an association between the role of NT on insulin releasing and glucose homeostasis (Grunddal et al. 2016). Furthermore, the distribution of NTR was found on plasma membranes of hepatocytes in male Wistar rats (Piatek et al. 2011). However, the activity of NT/NTR on hepatic insulin sensitivity is not thoroughly understood. The expression and function of NTR pertinent to hepatic insulin pathway under normal and pre-diabetic conditions requires further clarification. In contrast to our results, a research by Ratner and colleagues indicated that there was no change in the levels of plasma insulin of mice fed with a

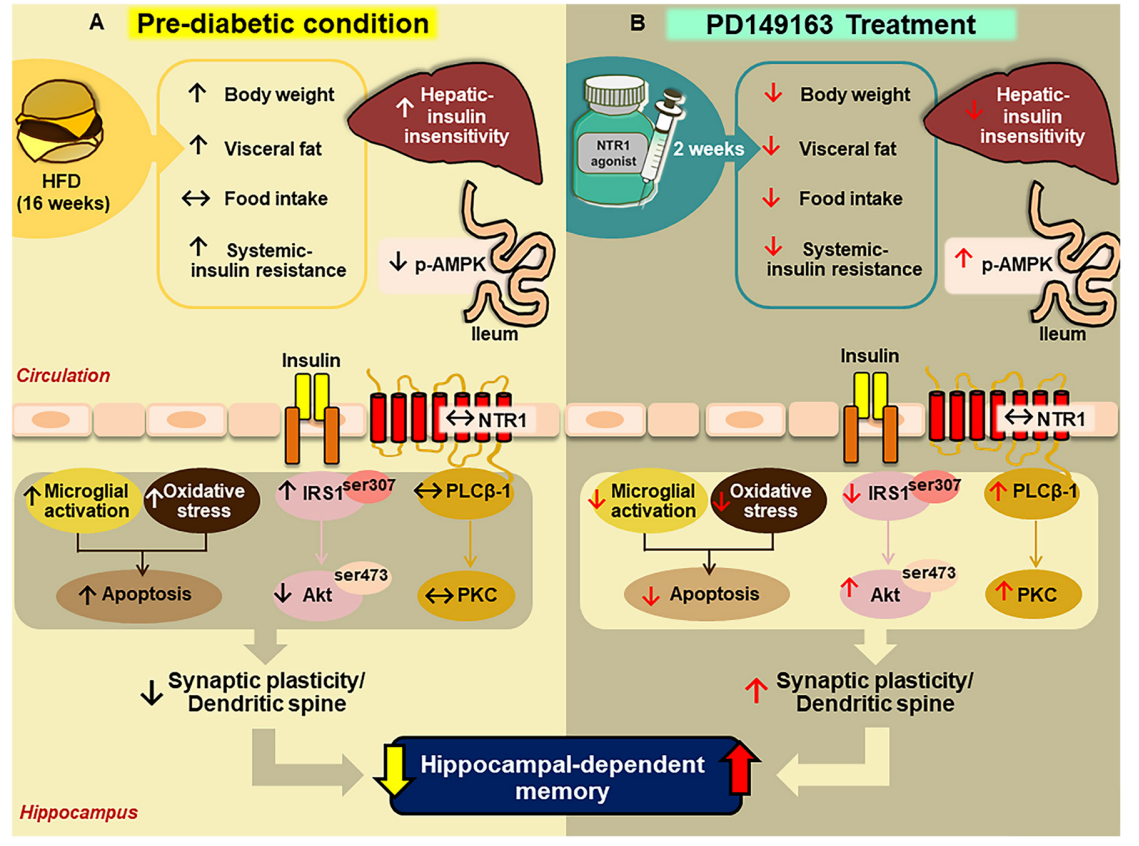

\section{Figure 10}

A schematic diagram represents the beneficial effects of NTR1 activator on peripheral and hippocampal pathologies in pre-diabetes. (A) HFD consumption for 16 weeks developed pre-diabetic condition by increasing body weight, visceral fat, hepatic/systemic insulin insensitivity as well as disrupted the activity of ileal NT-related protein (p-AMPK). It also enhanced the hippocampal pathologies including microglia hyper-activation, oxidative stress, apoptotic cell death, insulin insensitivity, synaptic dysplasticity, and dendritic spine loss, resulting in hippocampal memory deficits. (B) The restoration of peripheral and hippocampal pathologies after treated with NTR1 agonist (PD149163) in pre-diabetic rats for 2 weeks, leading to improve hippocampal learning and memory. HFD, high-fat diet; IRS1, insulin receptor substrate $1 ; \mathrm{PKC}$, protein kinase $\mathrm{C}$; PLC $\beta-1$, phospholipase $C$ beta-1. A full color version of this figure is available at https://doi.org /10.1530/JOE-20-0439. https://joe.bioscientifica.com https://doi.org/10.1530/JOE-20-0439
(C) 2021 Society for Endocrinology Published by Bioscientifica Ltd.
Printed in Great Britain 
high-fat, high-sucrose diet and treated with pegylated-NT (NT analog) for 6 days (Ratner et al. 2019). This might be due to a difference in the type of NT analog. Additionally, the effects of the combined treatment in HFD-fed group on peripheral metabolic parameters followed a similar pattern to those observed in HFD-fed rats treated with the NTR1 agonist. These results could be due to the differential expression of NTR1, as well as other subtypes of NTR, in different organs. Further clarification is needed regarding the expression of NTR subtypes and the mechanistic link of the NTR1 agonist/antagonist in the control of metabolic parameters in pre-diabetic conditions. Collectively, all findings show that the activation of NTR1 has beneficial effects on controlling metabolic disturbance in the prediabetic model. In the current study, even though the levels of fasting blood glucose and insulin in our HFD-fed control groups seem high, they are within the pre-diabetic ranges as consistent with previous studies (Chunchai et al. 2018, Jinawong et al. 2020, Konda et al. 2020). Meanwhile, the levels of these parameters in our ND-fed control are similar to the diet-control group in a previous study, which did not develop the pre-diabetic condition (Moreno-Fernandez et al. 2018). In addition, the rats in our study at the stage of experimentation were 18-20 weeks old, which is considered to be in early-to-mid adulthood (Sengupta 2013, Ohmura \& Kuniyoshi 2017).

According to our findings, treatment with the NTR1 activator had favorable effects on the periphery. Therefore, it might be used to ameliorate the hippocampal pathologies and cognitive decline after chronic HFD intake. A previous study has revealed that an NT/NTR1 activator reversed the impairment of spatial learning and memory via PLC $\beta-1$ and PKC signaling in the entorhinal cortex of APP/PS1 mice (Xiao et al. 2014). Although HFD did not alter the expression of PLC $\beta-1$ and PKC in this study, exogenous NTR1 agonist treatment increased their expression in the hippocampus, which were reversed after treatment with the NTR antagonist or combined therapy in HFDfed group. The findings of the current study demonstrate that the neuroprotective effects of the NTR1 agonist were mediated, at least partially, through the hippocampal PLC $\beta-1 /$ PKC pathway. According to previous evidence, NTR1 stimulation showed synergistic effects with GLP-1R activation on the reduction of metabolic dysfunction via the hypothalamic melanocortin pathway (Ratner et al. 2019). It also improved brain insulin signaling by enhancing the levels of GLP-1R, mTOR, and Erk2 genes in HFD-fed mice (Denver et al. 2018). These findings propose that the activation of NTR1 is involved with brain insulin signaling. Hippocampal insulin insensitivity, as shown by an elevation in the ratio of p-IRS1 $1^{\mathrm{ser} 307 / I R S 1}$ and reduction in the ratio of $\mathrm{p}-\mathrm{Akt} \mathrm{t}^{\mathrm{ser}} \mathrm{s07} / \mathrm{Akt}$ after incubation with insulin, was found in HFD-fed group and it was restored following treatment with the NTR1 agonist in the present study. The treatment with the NTR antagonist and combined therapy showed similar results to the HFD-fed rats treated with vehicle. Altogether, these results speculate that the benefits of NTR1 activation in the hippocampus are possibly mediated through the modulation of hippocampal insulin signaling. However, the insight into the mechanisms occurring between NTR1 and insulin signaling in the hippocampal pathologies under pre-diabetic conditions is still limited. Therefore, the molecular mechanisms of these pathways using NTR1- or insulin-related genes/ proteins depletion on hippocampal pathologies in the pre-diabetic model should be examined in the future.

Excessive fat consumption and insulin resistance in both the periphery and the hippocampus trigger hippocampal pathologies which in turn cause hippocampal synaptic dysfunction and memory deficits (Stranahan et al. 2008, Chunchai et al. 2019). Our findings were consistent with these studies. The hippocampal synaptic dysfunction and spine loss occurred and were restored within normal limits after NTR1 agonist treatment. To support these findings, Zhang and colleagues indicated that the transmission of neurotransmitters, particularly glutamate, occurred after NTR1 administration, leading to increased excitatory postsynaptic currents in AMPA receptors. As a result, neuronal synaptic plasticity was enhanced through the perforant pathway (Zhang et al. 2015). Furthermore, the excitation of neurons in the entorhinal cortex was observed following activation of NTR1 (Xiao et al. 2014). Overall, these effects of NTR1 activation led to the amelioration of synaptic dysfunction and dendritic spine loss, resulting in the improvement of cognitive function.

Previous evidences have revealed that NTR1 activation is negatively associated with brain inflammation and apoptosis (Denver et al. 2018, Zhao et al. 2020). The increased Iba-1 positive cells were detected and indicated an inflammatory response in cases of obesity as described in previous reports (Chunchai et al. 2018, Cope et al. 2018). Treatment with an NTR1 agonist decreased the number of microglial cells, resulting in diminished levels of pro-inflammatory cytokines and inflammation in animal models (Denver et al. 2018). Apart from this parameter, the brain apoptosis, as shown by enhancing the number of TUNEL positive cells and the level of Bax/Bcl-2, was detected in stroke model which could be reversed by the application of an NTR1 agonist (Zhao 
et al. 2020). Treatment with modified xenin-25, which can activate NTR1 in HFD-fed mice, led to the reduction of oxidative stress by alleviating the level of 8-oxoguanine in the dentate gyrus (Denver et al. 2018). Therefore, NTR1 stimulation diminished the levels of brain oxidative stress and apoptosis. Likewise, our findings demonstrated that treatment with an NTR1 agonist alleviated the number of Iba-1 positive cells, the levels of ROS production, TUNEL positive cells, and Bax/Bcl-2 ratio in the hippocampus. The attenuation of these hippocampal pathologies resulted in an improvement in neuronal synaptic plasticity and cognition (Sa-Nguanmoo et al. 2018).

Surprisingly, treatment with NTR1 activator not only restored the peripheral/hippocampal pathologies but also ameliorated cognitive decline in the HFD-fed group, which were abolished after treated with the NTR antagonist and combined therapy. To add weight to our findings, the advantageous effects of NT/NTR1 injections on memory restoration have been presented in various animal models. The results have demonstrated that an intra-cerebroventricular injection of NT improved cognition in male Sprague-Dawley rats, male Wistar rats, and male Lister hooded rats via NTR1 activation (Azmi et al. 2006, Laszlo et al. 2010, Xiao et al. 2014). The s.c. injection of NTR1 activator also restored the impairment of spatial memory in Brown-Norway rats (Keiser et al. 2014). Additionally, a previous clinical study reported a relationship between the expression of NTR1 genes (rs433545, rs6090435) and working memory tasks (Li et al. 2011). Apart from these conditions, the injection of NTR1 agonist alleviated cognitive decline in the Alzheimer's model (Xiao et al. 2014). Therefore, the activation of NTR1 improves learning and memory processes.

Previous evidence demonstrated that the models of HFD-induced pre-diabetes and Alzheimer's disease (AD) caused defect of episodic, hippocampal-dependent memory (Pratchayasakul et al. 2011, Underwood \& Thompson 2016, Setti et al. 2017). However, there are some differences between these two models. AD is the source of dementia with two major hallmarks, which are the aggregation of amyloid beta $(A \beta)$ plaque outside and between the neurons and the presence of neurofibrillary tangle inside the neurons (de Paula et al. 2009). The emergence of these hallmarks may be genetically determined (familial $\mathrm{AD}$ ) or arise from the interplay among multiple factors such as aging, metabolic abnormalities, and sedentary lifestyle (de Paula et al. 2009). The neuropathologic findings in AD models include neuro-inflammation, mitophagic impairment, oxidative stress, and neuronal cell loss (Mizuno et al. 2012,
Fuger et al. 2017, Fang et al. 2019). On the other hand, the consumption of HFD not only enhances the peripheral pathologies (gut dysbiosis, metabolic dysfunction, inflammation, and oxidative stress) but also triggers the hippocampal/brain pathologies including inflammation, insulin insensitivity, neuronal cell death, and neuronal synaptic dysplasticity which lead to learning and memory loss (Thiennimitr et al. 2018, Tan \& Norhaizan 2019, Jinawong et al. 2020). Notably, long-term of HFD consumption led to a high level of brain $\mathrm{A} \beta$ and the expression of Alzheimer's-related proteins (Saiyasit et al. 2019, Arunsak et al. 2020). Hence, AD and obese-insulin resistance-induced cognitive dysfunction are different in terms of the neuromolecular hallmarks and the upstream pathophysiological mechanisms but features in the larger scales and the downstream outcomes (e.g. neuronal cell loss and functional deterioration) are similar.

In the current study, the NTR1 activator exerted advantageous effects via the restoration of metabolic disturbances, hippocampal pathologies, and cognitive deficits through the modulation of hippocampal NTR1 and insulin signaling. Nevertheless, the administration of NTR1 agonist-NTR antagonist combination led to discrepant results in the brain and in the peripheral tissues, which could be attributed to several plausible explanations. First, there is an organ-specific variation of NTR expression (Boudin et al. 1996, Rowe et al. 2006, Piatek et al. 2011). This could be exemplified by the fact that NTR1 was upregulated and its downstream signaling molecule, p-AMPK, was downregulated by HFD in the ileum but not in the hippocampus. Secondly, the dose of the NTR antagonist used in this study may not be sufficient to produce peripheral responses. There are previous studies in rats demonstrating that the s.c. injection or an intra-peritoneal administration of SR142948A at the dose of $1 \mathrm{mg} / \mathrm{kg}$ efficiently blocked the NTR1 activity in the brain (Azmi et al. 2006, Keiser et al. 2014). The effective doses for NTR1 blockade in peripheral organs, however, are yet to be defined. Thirdly, the drugs may be distributed inhomogeneously in the body, leading to different drug concentrations in various tissues. Unfortunately, such data regarding the tissue distribution of those drugs used in our study are still lacking. Therefore, to support or refute these hypotheses, investigations that take into account such as (1) the organ-specific NTR expression levels, (2) dose-dependent effects of the drugs that modulate NTR signaling in various tissues, and (3) detailed pharmacokinetic/pharmacodynamic studies of the drugs should be conducted in the future. Aside from the aforementioned parameters, future study may also 
assess the effect of NTR1 modulators on body temperature in HFD-induced pre-diabetic rats.

In summary, the activation of NTR1 attenuates metabolic impairment and exerts protective effects in the hippocampus, resulting in the improvement of hippocampal-dependent memory following chronic HFD consumption. These results propose that NTR1 agonist may be used as a therapeutic approach to prevent metabolic dysfunction, hippocampal pathologies, and memory decline in patients with obesity and the prediabetic condition.

\section{Supplementary materials}

This is linked to the online version of the paper at https://doi.org/10.1530/ JOE-20-0439.

\section{Declaration of interest}

The authors declare that there is no conflict of interest that could be perceived as prejudicing the impartiality of the research reported.

\section{Funding}

The present project was supported by the Senior Research Scholar Grant from the National Research Council of Thailand, Thailand (S C C); the Royal Golden Jubilee Ph.D. program, Thailand (PHD/0111/2559 N S and S C C); an NSTDA Research Chair Grant from the National Science and Technology Development Agency, Thailand (N C) and a Chiang Mai University Excellence Center Award, Thailand (N C)

\section{References}

Arunsak B, Pratchayasakul W, Amput P, Chattipakorn K, Tosukhowong T, Kerdphoo S, Jaiwongkum T, Thonusin C, Palee S, Chattipakorn N, et al. 2020 Proprotein convertase subtilisin/kexin type 9 (PCSK9) inhibitor exerts greater efficacy than atorvastatin on improvement of brain function and cognition in obese rats. Archives of Biochemistry and Biophysics 689 108470. (https://doi.org/10.1016/j. abb.2020.108470)

Azmi N, Norman C, Spicer CH \& Bennett GW 2006 Effects of a neurotensin analogue (PD149163) and antagonist (SR142948A) on the scopolamine-induced deficits in a novel object discrimination task. Behavioural Pharmacology 17 357-362. (https://doi. org/10.1097/01.fbp.0000224382.63744.20)

Boudin H, Pelaprat D, Rostene W \& Beaudet A 1996 Cellular distribution of neurotensin receptors in rat brain: immunohistochemical study using an antipeptide antibody against the cloned high affinity receptor. Journal of Comparative Neurology 373 76-89. (https:// doi.org/10.1002/(SICI)1096-9861(19960909)373:1<76::AIDCNE7>3.0.CO;2-A)

Candan N \& Tuzmen N 2008 Very rapid quantification of malondialdehyde (MDA) in rat brain exposed to lead, aluminium and phenolic antioxidants by high-performance liquid chromatographyfluorescence detection. Neurotoxicology 29 708-713. (https://doi. org/10.1016/j.neuro.2008.04.012)
Carraway R \& Leeman SE 1973 The isolation of a new hypotensive peptide, neurotensin, from bovine hypothalami. Journal of Biological Chemistry 248 6854-6861.

Chunchai T, Thunapong W, Yasom S, Wanchai K, Eaimworawuthikul S, Metzler G, Lungkaphin A, Pongchaidecha A, Sirilun S, Chaiyasut C, et al. 2018 Decreased microglial activation through gut-brain axis by prebiotics, probiotics, or synbiotics effectively restored cognitive function in obese-insulin resistant rats. Journal of Neuroinflammation 15 11. (https://doi.org/10.1186/s12974-018-1055-2)

Chunchai T, Keawtep P, Arinno A, Saiyasit N, Prus D, Apaijai N, Pratchayasakul W, Chattipakorn N \& Chattipakorn SC 2019 N-acetyl cysteine, inulin and the two as a combined therapy ameliorate cognitive decline in testosterone-deprived rats. Aging 11 3445-3462. (https://doi.org/10.18632/aging.101989)

Cope EC, LaMarca EA, Monari PK, Olson LB, Martinez S, Zych AD, Katchur NJ \& Gould E 2018 Microglia play an active role in obesityassociated cognitive decline. Journal of Neuroscience 38 8889-8904. (https://doi.org/10.1523/JNEUROSCI.0789-18.2018)

de Paula VJR, Guimaraes FM, Diniz BS \& Forlenza OV 2009 Neurobiological pathways to Alzheimer's disease: amyloid-beta, tau protein or both? Dementia and Neuropsychologia 3 188-194. (https:// doi.org/10.1590/S1980-57642009DN30300003)

Denenberg VH 1969 Open-field bheavior in the rat: what does it mean? Annals of the New York Academy of Sciences 159 852-859. (https://doi. org/10.1111/j.1749-6632.1969.tb12983.x)

Denver P, Gault VA \& McClean PL 2018 Sustained high-fat diet modulates inflammation, insulin signalling and cognition in mice and a modified xenin peptide ameliorates neuropathology in a chronic high-fat model. Diabetes, Obesity and Metabolism 20 1166-1175. (https://doi.org/10.1111/dom.13210)

Fang EF, Hou Y, Palikaras K, Adriaanse BA, Kerr JS, Yang B, Lautrup S, Hasan-Olive MM, Caponio D, Dan X, et al. 2019 Mitophagy inhibits amyloid-beta and tau pathology and reverses cognitive deficits in models of Alzheimer's disease. Nature Neuroscience 22 401-412. (https://doi.org/10.1038/s41593-018-0332-9)

Fazzari G, Zizza M, Di Vito A, Alò R, Mele M, Bruno R, Barni T, Facciolo RM \& Canonaco M 2018 Reduced learning and memory performances in high-fat treated hamsters related to brain neurotensin receptor1 expression variations. Behavioural Brain Research 347 227-233. (https://doi.org/10.1016/j.bbr.2018.03.015)

Feifel D, Goldenberg J, Melendez G \& Shilling PD 2010 The acute and subchronic effects of a brain-penetrating, neurotensin-1 receptor agonist on feeding, body weight and temperature. Neuropharmacology 58 195-198. (https://doi.org/10.1016/j.neuropharm.2009.07.001)

Fuger P, Hefendehl JK, Veeraraghavalu K, Wendeln AC, Schlosser C, Obermuller U, Wegenast-Braun BM, Neher JJ, Martus P, Kohsaka S, et al. 2017 Microglia turnover with aging and in an Alzheimer's model via long-term in vivo single-cell imaging. Nature Neuroscience 20 1371-1376. (https://doi.org/10.1038/nn.4631)

Grunddal KV, Ratner CF, Svendsen B, Sommer F, Engelstoft MS, Madsen AN, Pedersen J, Nohr MK, Egerod KL, Nawrocki AR, et al. 2016 Neurotensin is coexpressed, coreleased, and acts together With GLP-1 and PYY in enteroendocrine control of metabolism. Endocrinology 157 176-194. (https://doi.org/10.1210/en.2015-1600)

Jansen KL, Faull RL, Dragunow M \& Synek BL 1990 Alzheimer's disease: changes in hippocampal N-methyl-D-aspartate, quisqualate, neurotensin, adenosine, benzodiazepine, serotonin and opioid receptors - an autoradiographic study. Neuroscience 39 613-627. (https://doi.org/10.1016/0306-4522(90)90246-z)

Jinawong K, Apaijai N, Wongsuchai S, Pratchayasakul W, Chattipakorn N \& Chattipakorn SC 2020 Necrostatin-1 mitigates cognitive dysfunction in prediabetic rats with no alteration in insulin sensitivity. Diabetes 69 1411-1423. (https://doi.org/10.2337/ db19-1128)

Keawtep P, Pratchayasakul W, Arinno A, Apaijai N, Chunchai T, Kerdphoo S, Jaiwongkum T, Chattipakorn N \& Chattipakorn SC 
2019 Combined dipeptidyl peptidase-4 inhibitor with low-dose testosterone exerts greater efficacy than monotherapy on improving brain function in orchiectomized obese rats. Experimental Gerontology 123 45-56. (https://doi.org/10.1016/j.exger.2019.05.008)

Keiser AA, Matazel KS, Esser MK, Feifel D \& Prus AJ 2014 Systemic administration of the neurotensin NTS(1)-receptor agonist PD149163 improves performance on a memory task in naturally deficient male brown Norway rats. Experimental and Clinical Psychopharmacology 22 541-547. (https://doi.org/10.1037/a0037912)

Konda PY, Poondla V, Jaiswal KK, Dasari S, Uyyala R, Surtineni VP, Egi JY, Masilamani AJA, Bestha L, Konanki S, et al. 2020 Pathophysiology of high fat diet induced obesity: impact of probiotic banana juice on obesity associated complications and hepatosteatosis. Scientific Reports 10 16894. (https://doi.org/10.1038/s41598-020-73670-4)

Kumfu S, Charununtakorn ST, Jaiwongkam T, Chattipakorn N \& Chattipakorn SC 2018 Humanin exerts neuroprotection during cardiac ischemia-reperfusion injury. Journal of Alzheimer's Disease $\mathbf{6 1}$ 1343-1353. (https://doi.org/10.3233/JAD-170708)

Laszlo K, Toth K, Kertes E, Peczely L, Ollmann T \& Lenard L 2010 Effects of neurotensin in amygdaloid spatial learning mechanisms. Behavioural Brain Research 210 280-283. (https://doi.org/10.1016/j. bbr.2010.02.038)

Li J, Chen C, Chen C, He Q, Li H, Li J, Moyzis RK, Xue G \& Dong Q 2011 Neurotensin receptor 1 gene (NTSR1) polymorphism is associated with working memory. PLOS ONE 6 e17365. (https://doi.org/10.1371/ journal.pone.0017365)

Li J, Song J, Zaytseva YY, Liu Y, Rychahou P, Jiang K, Starr ME, Kim JT, Harris JW, Yiannikouris FB, et al. 2016 An obligatory role for neurotensin in high-fat-diet-induced obesity. Nature 533 411-415. (https://doi.org/10.1038/nature17662)

Mayans L 2015 Metabolic syndrome: insulin resistance and prediabetes. FP Essentials 435 11-16.

McGillicuddy FC, Reynolds CM, Finucane O, Coleman E, Harford KA, Grant C, Sergi D, Williams LM, Mills KH \& Roche HM 2013 Longterm exposure to a high-fat diet results in the development of glucose intolerance and insulin resistance in interleukin-1 receptor I-deficient mice. American Journal of Physiology: Endocrinology and Metabolism 305 E834-E844. (https://doi.org/10.1152/ajpendo.00297.2013)

Mizuno S, Iijima R, Ogishima S, Kikuchi M, Matsuoka Y, Ghosh S, Miyamoto T, Miyashita A, Kuwano R \& Tanaka H 2012 AlzPathway: a comprehensive map of signaling pathways of Alzheimer's disease. BMC Systems Biology 6 52. (https://doi.org/10.1186/1752-0509-6-52)

Moreno-Fernandez S, Garces-Rimon M, Vera G, Astier J, Landrier JF \& Miguel M 2018 High fat/high glucose diet induces metabolic syndrome in an experimental rat model. Nutrients 10 1502. (https:// doi.org/10.3390/nu10101502)

Nguyen DM \& El-Serag HB 2010 The epidemiology of obesity. Gastroenterology Clinics of North America 39 1-7. (https://doi. org/10.1016/j.gtc.2009.12.014)

Ohmura Y \& Kuniyoshi Y 2017 A translational model to determine rodent's age from human foetal age. Scientific Reports 717248. (https://doi.org/10.1038/s41598-017-17571-z)

Piatek J, Mackowiak P, Krauss H, Nowak D \& Bogdanski P 2011 In vivo investigations of neurotensin receptors in adipocytes, hepatocytes and enterocytes of rat. Annals of Agricultural and Environmental Medicine 18 433-436.

Pintana H, Pongkan W, Pratchayasakul W, Chattipakorn N \& Chattipakorn SC 2015 Testosterone replacement attenuates cognitive decline in testosterone-deprived lean rats, but not in obese rats, by mitigating brain oxidative stress. Age 37 84. (https://doi.org/10.1007/ s11357-015-9827-4)

Pintana H, Tanajak P, Pratchayasakul W, Sa-Nguanmoo P, Chunchai T, Satjaritanun P, Leelarphat L, Chattipakorn N \& Chattipakorn SC 2016 Energy restriction combined with dipeptidyl peptidase-4 inhibitor exerts neuroprotection in obese male rats. British Journal of Nutrition 1-9. (https://doi.org/10.1017/S0007114516003871)
Pratchayasakul W, Kerdphoo S, Petsophonsakul P, Pongchaidecha A, Chattipakorn N \& Chattipakorn SC 2011 Effects of high-fat diet on insulin receptor function in rat hippocampus and the level of neuronal corticosterone. Life Sciences 88 619-627. (https://doi. org/10.1016/j.lfs.2011.02.003)

Pratchayasakul W, Chattipakorn N \& Chattipakorn SC 2014 Estrogen restores brain insulin sensitivity in ovariectomized non-obese rats, but not in ovariectomized obese rats. Metabolism: Clinical and Experimental 63 851-859. (https://doi.org/10.1016/j.metabol.2014.03.009)

Ratner C, He Z, Grunddal KV, Skov LJ, Hartmann B, Zhang F, Feuchtinger A, Bjerregaard A, Christoffersen C, Tschöp MH, et al. 2019 Long-acting neurotensin synergizes with liraglutide to reverse obesity through a melanocortin-dependent pathway. Diabetes 68 1329-1340. (https://doi.org/10.2337/db18-1009)

Rollins CPE, Gallino D, Kong V, Ayranci G, Devenyi GA, Germann J \& Chakravarty MM 2019 Contributions of a high-fat diet to Alzheimer's disease-related decline: a longitudinal behavioural and structural neuroimaging study in mouse models. NeuroImage. Clinical 21 101606. (https://doi.org/10.1016/j.nicl.2018.11.016)

Rowe WB, Kar S, Meaney MJ \& Quirion R 2006 Neurotensin receptor levels as a function of brain aging and cognitive performance in the Morris water maze task in the rat. Peptides 27 2415-2423. (https://doi. org/10.1016/j.peptides.2006.03.036)

Sahu A 2008 Effects of chronic central leptin infusion on proopiomelanocortin and neurotensin gene expression in the rat hypothalamus. Neuroscience Letters 440 125-129. (https://doi. org/10.1016/j.neulet.2008.05.083)

Saiyasit N, Chunchai T, Prus D, Suparan K, Pittayapong P, Apaijai N, Pratchayasakul W, Sripetchwandee J, Chattipakorn MDPDN \& Chattipakorn SC 2019 Gut dysbiosis develops before metabolic disturbance and cognitive decline in high-fat diet-induced obese condition. Nutrition 69110576.

Saiyasit N, Chunchai T, Apaijai N, Pratchayasakul W, Sripetchwandee J, Chattipakorn N \& Chattipakorn SC 2020 Chronic high-fat diet consumption induces an alteration in plasma/brain neurotensin signaling, metabolic disturbance, systemic inflammation/oxidative stress, brain apoptosis, and dendritic spine loss. Neuropeptides $\mathbf{8 2}$ 102047. (https://doi.org/10.1016/j.npep.2020.102047)

Sa-Nguanmoo P, Tanajak P, Kerdphoo S, Jaiwongkam T, Wang X, Liang G, Li X, Jiang C, Pratchayasakul W, Chattipakorn N, et al. 2018 FGF21 and DPP-4 inhibitor equally prevents cognitive decline in obese rats. Biomedicine and Pharmacotherapy 97 1663-1672. (https://doi. org/10.1016/j.biopha.2017.12.021)

Satoh K \& Matsumura H 1990 Distribution of neurotensin-containing fibers in the frontal cortex of the macaque monkey. Journal of Comparative Neurology 298 215-223. (https://doi.org/10.1002/ cne.902980206)

Sengupta P 2013 The laboratory rat: relating its age with human's. International Journal of Preventive Medicine 4 624-630.

Setti SE, Hunsberger HC \& Reed MN 2017 Alterations in hippocampal activity and Alzheimer's disease. Translational Issues in Psychological Science 3 348-356. (https://doi.org/10.1037/tps0000124)

Shrager Y, Bayley PJ, Bontempi B, Hopkins RO \& Squire LR 2007 Spatial memory and the human hippocampus. PNAS 104 2961-2966. (https://doi.org/10.1073/pnas.0611233104)

Stranahan AM, Norman ED, Lee K, Cutler RG, Telljohann RS, Egan JM \& Mattson MP 2008 Diet-induced insulin resistance impairs hippocampal synaptic plasticity and cognition in middle-aged rats. Hippocampus 18 1085-1088. (https://doi.org/10.1002/hipo.20470)

Tan BL \& Norhaizan ME 2019 Effect of high-fat diets on oxidative stress, cellular inflammatory response and cognitive function. Nutrients $\mathbf{1 1}$ 2579. (https://doi.org/10.3390/nu11112579)

Tanajak P, Pintana H, Siri-Angkul N, Khamseekaew J, Apaijai N, Chattipakorn SC \& Chattipakorn N 2017 Vildagliptin and caloric restriction for cardioprotection in pre-diabetic rats. Journal of Endocrinology 232 189-204. (https://doi.org/10.1530/JOE-16-0406) https://joe.bioscientifica.com

https://doi.org/10.1530/JOE-20-0439 (c) 2021 Society for Endocrinology Published by Bioscientifica Ltd. Printed in Great Britain 
Thiennimitr P, Yasom S, Tunapong W, Chunchai T, Wanchai K, Pongchaidecha A, Lungkaphin A, Sirilun S, Chaiyasut C, Chattipakorn N, et al. 2018 Lactobacillus paracasei HII01, xylooligosaccharides, and synbiotics reduce gut disturbance in obese rats. Nutrition 54 40-47. (https://doi.org/10.1016/j. nut.2018.03.005)

Thonusin C, Apaijai N, Jaiwongkam T, Kerdphoo S, Arunsak B, Amput P, Palee S, Pratchayasakul W, Chattipakorn N \& Chattipakorn SC 2019 The comparative effects of high dose atorvastatin and proprotein convertase subtilisin/kexin type 9 inhibitor on the mitochondria of oxidative muscle fibers in obese-insulin resistant female rats. Toxicology and Applied Pharmacology 382 114741. (https://doi. org/10.1016/j.taap.2019.114741)

Underwood EL \& Thompson LT 2016 A high-fat diet causes impairment in hippocampal memory and sex-dependent alterations in peripheral metabolism. Neural Plasticity 2016 7385314. (https://doi. org/10.1155/2016/7385314)

Vincent JP 1995 Neurotensin receptors: binding properties, transduction pathways, and structure. Cellular and Molecular Neurobiology 15 501-512. (https://doi.org/10.1007/BF02071313)

Vorhees CV \& Williams MT 2006 Morris water maze: procedures for assessing spatial and related forms of learning and memory.
Nature Protocols 1 848-858. (https://doi.org/10.1038/ nprot.2006.116)

Xiao Z, Cilz NI, Kurada L, Hu B, Yang C, Wada E, Combs CK, Porter JE, Lesage F \& Lei S 2014 Activation of neurotensin receptor 1 facilitates neuronal excitability and spatial learning and memory in the entorhinal cortex: beneficial actions in an Alzheimer's disease model. Journal of Neuroscience $\mathbf{3 4}$ 7027-7042. (https://doi.org/10.1523/ JNEUROSCI.0408-14.2014)

Yang RL, Shi YH, Hao G, Li W \& Le GW 2008 Increasing oxidative stress with progressive hyperlipidemia in human: relation between malondialdehyde and atherogenic index. Journal of Clinical Biochemistry and Nutrition 43 154-158. (https://doi.org/10.3164/ jcbn.2008044)

Zhang H, Dong H \& Lei S 2015 Neurotensinergic augmentation of glutamate release at the perforant path-granule cell synapse in rat dentate gyrus: roles of L-type Ca(2). Neuropharmacology 95 252-260. (https://doi.org/10.1016/j.neuropharm.2015.03.028)

Zhao Y, Wei ZZ, Lee JH, Gu X, Sun J, Dix TA, Wei L \& Yu SP 2020 Pharmacological hypothermia induced neurovascular protection after severe stroke of transient middle cerebral artery occlusion in mice. Experimental Neurology 325 113133. (https://doi.org/10.1016/j. expneurol.2019.113133)

Received in final form 17 October 2020

Accepted 26 October 2020

Accepted Manuscript published online 26 October 2020
(C) 2021 Society for Endocrinology Published by Bioscientifica Ltd. 